\title{
Simulation Based Decision Support System for Cut-to-size Plants
}

\author{
Robert Schoech \\ Susanne Schmid \\ Christian Hillbrand \\ Industrial Research and Development \\ V-Research GmbH \\ Stadtstrasse 33, 6850 Dornbirn, Austria \\ E-mail: robert.schoech@v-research.at, susanne.schmid@v-research.at, christian.hillbrand@v-research.at
}

\section{KEYWORDS}

Discrete Event Simulation, DEVS, Emulation, Central Hierarchical Control Systems, Decision Support Tool, Cut-to-size Plants, Plant Engineering

\begin{abstract}
Cut-to-size plants are very complex systems, which are used to process several kinds of panels. The acquisition, planning, implementation and operation of these systems impose lots of challenges. To support the process from retail to operation of cut-to-size plants, a new simulation based decision support tool has been developed. This planning tool offers entirely new opportunities for system experts to model, animate, simulate and emulate cut-to-size processes. In this paper, we discuss centrally and hierarchically controlled real time systems and derive a conceptual design to explain the implemented reproduction of the virtual system. We introduce the system architecture for our simulation based decision support tool and examine our approach to model largescale plant systems. Apart from this, we present our method to process the orders which are generated on the control system and to handle them as discrete event tasks in the virtual system. Finally, based on the findings of the realization and testing of the tool, this paper discusses the opportunities arising from this approach as well as its future potential.
\end{abstract}

\section{INTRODUCTION}

Cut-to-size plants with sorting and stacking solutions are very complex systems. Some of the characteristic problems are parallel material movement, buffer-areas and a large number of simultaneous activities. On cut-tosize plants several kinds of panels can be processed. The main cut-to-size processes are cutting, sorting and stacking. These processes are executed on machines like cut-to-size saws, sorting carriages, stacking devices, roller tracks, etc. In combination with their control unit, these machines are specified as self-contained plant units.

The acquisition, planning, implementation and operation of cut-to-size plants impose special challenges. Some of these can be traced back to the fact that a sales process usually takes place before the initiation of planning and implementation, since cut-to-size plants are built according to customer specifications (make-to-order strategy). Plant businesses show a high level of specialization of its product range and services (Pekrul 2006). In case of cut-to-size plants, performance is usually measured by cycle times. Besides that, there usually exist substantial information and knowledge asymmetries between suppliers and customers (Pekrul 2006). As the latter is not capable of evaluating the whole complexity of a plant, he needs to put a lot of confidence into the provider's projections concerning the performance and benefits of the plant.

An essential sales instrument of cut-to-size plants is the reputation of the provider company. Apart from this, reference plants are used to prove the technical feasibility and efficiency of the plants to the customers (Soellner 2008). This is one of the reasons why the marketing of plants is a very complex organizational process and can be viewed as an acquisition process which contains a considerable amount of risk for both parties. The supplier usually faces substantial sunk costs if the customer decides not to purchase the plant upon a rather extensive projection process. In order to reduce supplier-side risks, this paper describes a simulationbased approach to support the process from retail to operation and to make the distribution of cut-to-size plants more efficient. This planning instrument makes it possible for system experts to model, animate, simulate and emulate the cut-to-size processes without a need for any deeper simulation or emulation knowledge.

Among others, one main challenge in building this decision support tool was the determination of the procedural and structural correlations which allowed the establishment of the hierarchy and the modularization of the plant- and control system modules and processes.

\section{THEORETICAL PRINCIPLES AND LITERATURE REVIEW}

Before providing details about the simulation approach for sales and projection processes, the following section will introduce some basic theoretical principles of simulation and emulation techniques. Based on that we will provide a review of the relevant literature within the field of discrete event simulation for decision support in industrial processes. 


\section{Discrete event simulation for supporting industrial process planning activities}

As it will be discussed below, simulation techniques are likely to provide a wide range of analytical possibilities for planning and evaluating industrial processes. In the context of this paper, a future manufacturing process as designed in the sales phase can be considered as one specific industrial process. In order apply analytical techniques to arbitrary decision or planning scenarios in industrial processes, it is necessary to map these problems to virtual models. The simpler and clearer the problem can be defined by means of a mathematical model, the more likely deterministic methods are implemented. If significant influences from uncertainty factors have to be assumed, stochastic techniques are to be used, and, provided the problem exceeds a certain degree of complexity, simulation methods will be implemented. The latter have the advantage, among other things, that they, on the one hand, can map the uncertainties associated to a process through stochastic influences, and, on the other hand, the dynamic behavior of processes in different scenarios can be evaluated without increasing the runtime of the evaluation algorithms dramatically. Since real models from an industrial environment tend to have a high degree of complexity, the latter characteristic of simulation techniques in particular facilitates their practical implementation in the supply chain in many cases (Min and Zhou 2002; Shah 2005).

Simulation is defined as a method to reproduce dynamic processes in a virtual model which makes it possible to analyze the behavior of complex systems (VDI 3633 2000). Especially for decision support and planning activities for industrial processes, the type of discrete event simulation has evolved as a key methodology (Kleijnen 2005). In discrete event simulation, the state of a model changes during the simulation at discrete points of time. The update of the simulation time occurs whenever an event is pending. A data structure containing events (usually an event list) is an integral part of the event discrete simulation engines and holds references for future events, ordered by time of occurrence (Banks et al. 2005). The realization of a simulation study is usually broken down into several steps as shown in figure 1.

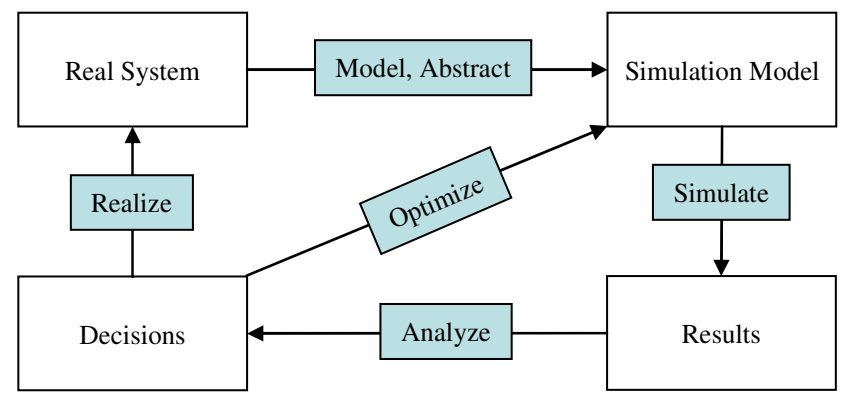

Figure 1 Steps of a simulation study
After the definition of the system and identification of the problem, the real system is transformed into an abstract simulation model. The simulation runs and the subsequent analysis of results can serve as an input for modifications of the model. Furthermore, the results of the simulated scenarios can be used to optimize and reanalyze the virtual model before applying changes to the real system.

As confirmed by numerous publications (Terzi and Cavalieri 2004, p. 5; Iannone et al. 2007, p. 222), specifically for the reasons mentioned above, discrete event simulation is one of the most popular decisionsupporting techniques in the area of process evaluation. For example, Chang and Makatsoris describe the advantages of discrete event simulation in the environment of Supply Chain Management as follows: "Discrete event simulation permits the evaluation of operating performance prior to the implementation of a system: It enables companies to perform powerful whatif analyses leading them to better planning decisions; it permits the comparison of various operational alternatives without interrupting the real system; it permits time compression so that timely policy decisions can be made" (Chang and Makatsoris 2001, p. 26). Hence, simulation techniques are employed within the scope of decision support mainly in the design phase (e.g. determining critical paths or bottle necks in the process).

In the literature, a variety of concepts and applications exist which are concerned with the application of discrete event simulation in Supply Chain Management (SCM). The application scenarios for this decisionsupporting method are widespread in this case. They include to a large extent the general optimization tasks which are to be performed by SCM (Archibald et al. 1999, p. 1207). The target variables of simulation studies concentrate mainly on a reduction of cost, the optimization of material and information flows, the reduction of processing and lead times, or a consequent process-related orientation of the company (Banks et al. 2002). A recent study analyzes over 80 articles which describe an application of Supply Chain Simulation either in an industrial pilot project, commercially available software, or a simulation test within a logistics chain (Terzi and Cavalieri 2004). This study shows that only 11 papers are concerned with manufacturing processes of which the vast majority aims at integrating the manufacturing process into the overall supply chain or scheduling of production lots. Only four papers describe scenarios for applying simulation for planning manufacturing layouts: Olhager and Persson describe the successful application of simulation for redesigning manufacturing plants in the electronics industry (Olhager and Persson 2006). The other three articles are related to the simulation software package Supply Chain Builder (SCB) of Simulation Dynamics Inc. (SDI). The first approaches of SDI concentrated primarily on the intra-organizational optimization of value chains. The Plant Builder, for example, is focused on the simulation 
of internal value chain activities (Siprelle et al. 1999). As an extension, the in-plant distribution as well as the supply chain on the distribution side is included in the simulation model (Phelbs et al. 2000; Phelbs et al. 2001).

Besides that, a number of articles report simulation studies in order to find optimal layouts for flexible manufacturing facilities (Drake et al. 1995; Zhou and Venkatesh 1999; Azadivar and Wang 2000; Aleisa and Lin 2005) or cellular manufacturing layouts (Morris and Tersine 1990; Irizarry et al. 2001). These approaches mostly aim at the (near) optimal solution of a specific problem rather than evaluating a multidimensional scenario like the assessment of feasibility for a future manufacturing plant projection.

By contrast, the starting point for optimizing the sales process is the definition of critical performance indicators (e.g. certain lead or cycle times) which have to be realizable by a projected facility or plant. Subsequently, the main issue is to identify admissible and feasible plant configurations which optimize these performance variables. As this paper will show, discrete event simulation can very efficiently support this task.

\section{Emulation based support for plant systems}

Emulation is the virtual reproduction of certain aspects of hardware or software systems (external system) on another system (host system) (Mertens 2006). Therefore emulation can be seen as a special case of simulation, supplemented with the coupling of real functional components. For emulation of cut-to-size plants, job data is generated within a "virtual plant" and processed by the simulation tool.

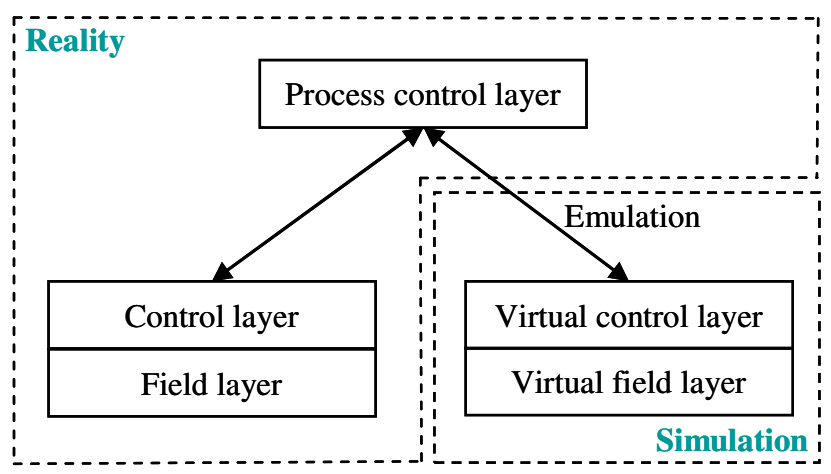

Figure 2 Central, hierarchical control systems

Classical plants are centrally and hierarchically controlled real time systems (see figure 2) (Guenthner and ten Hompel 2010). On a real system the field layer, which constitutes the lowest level, represents all mechanical components with their actuators and sensors and controls the material handling. The control layer resides above the process level. On this layer, sensor data is processed and control signals for the actuators are generated. This level represents the basis for an automated, unit based plant. Moreover, it coordinates the handover of load data and controls the material flow of the plant units. The process control layer is the highest layer in the control pyramid and is usually called plant server. The control layer and the process control layer are connected by means of a communication channel, which passes on the scheduled orders to the control layer and receives confirmation when all actions have been processed.

The plant logic of the real time system as described above is mapped to a model within the virtual plant: The virtual field layer of the emulated system visualizes all mechanical components and displays the kinematic movement of the material handling in a virtual view. The virtual control layer prepares all orders from the process control layer and controls each virtual plant unit. Since there is a tight coupling between real time and simulation systems this system can be viewed as an emulation system (Mc Gregor 2002).

\section{GENERAL DESIGN PRINCIPLES}

The following chapter provides a general overview of the system architecture for our simulation-based decision support approach. Also, the modeling process of the decision support system and the clear separation of virtual control layer and virtual field layer for the simulation model are discussed. Furthermore, our task handling method for event lists will be described.

\section{System architecture}

The kernel of our simulation based decision support system is based on an existing generic application platform. The latter has been implemented adhering to the principles of flexible three-tier architecture (Balzert 1999), consisting of the following layers:

- Database Layer,

- Application Layer and

- Graphical User Interface Layer (GUI).

The flexibility of the GUI is realized by a separation between the Application Layer and the Database Layer. Therefore a better performance is obtained for data visualization.

The application platform provides simulation libraries with generic modeling functions, which can be used to implement domain-specific modeling environments. A number of domain-specific modeling methods and applications, such as planning of transportation networks or warehousing structures, have already been implemented upon this platform (Dobler et al. 2008; Maerz and Saler 2008). As one part of the described platform, specific methods for cut-to-size plants were implemented. The main intention has been to enable domain experts (i.e. technical sales personnel) to define alternative projected plant configurations within the virtual system and then to evaluate them according to the predefined critical performance indicators in order to identify an optimal solution. By using a domain-specific modeling environment with an underlying simulation model built-in, the domain expert is used to take 
advantage of emulation techniques without the need for simulation expertise.

Therefore, the software architecture of the decision support system for cut-to-size-plants is arranged in two levels (see figure 3 ).

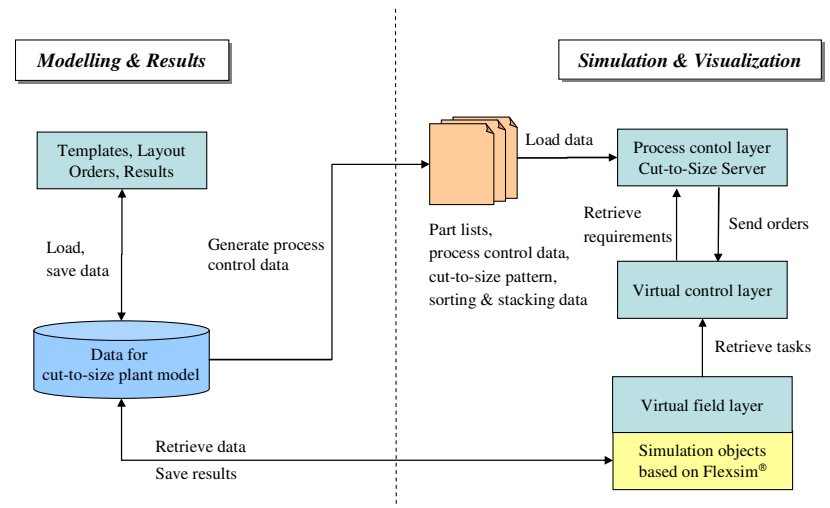

Figure 3 Software architecture for decision support system

The Modeling and Results Level is used for the development of unit-based models (Zeigler and Sarjoughian 2003) in which a plant is designed as a collection of plant units. Templates provide a set of unit based components and allow the specification of different types of models, depending on the goal of the modeler. The work flow of the plant is modeled implicitly by the coupling of the individual plant units. The modeled plant is persisted and process control data for the cut-to-size server is generated automatically. Depending on process control data, bills of materials, batch sizes, optimized cut-to-size patterns, sorting and stacking patterns for panels are then computed. The computation results represent the planning data which are used as a basis to generate the orders for the virtual plant. Furthermore, the simulation run and the visualization of the material flow are triggered at this level. Out of the orders, which are broadcasted by the cut-to-size server, task sequences are created (see figure 4).

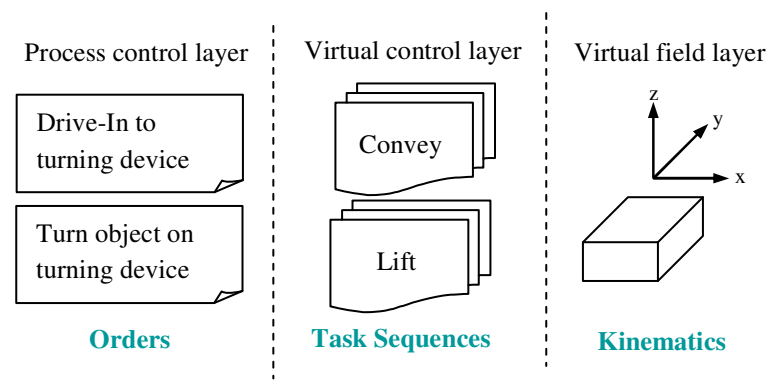

Figure 4 Order handling

A single task describes an activity that imitates the physical mechanism which is executed on the simulation object. An example for an activity is the turning of the panel with the device turning gear on the unit turning device. After the successful completion of the emulation run, the results, which are based on the recorded data, are ready for detailed analysis.

The Simulation and Visualization Level represents the virtual system, which is controlled. It is based on the simulation engine Flexsim( $\odot$. Depending on the model data and associated meta-information, the simulation model is created automatically. After the initialization and start of the emulation model, the virtual field layer receives the task sequences, which are created on the virtual control layer and transforms them into discrete events. According to these tasks, kinematic flows are created (see figure 4) and run time information is logged simultaneously. The received task sequences depend on bills of materials, process control data, cut-to-size patterns as well as sorting and stacking data.

\section{Modeling process}

Our approach to model large-scale plant systems is based on three complementary services called plant unit template, unit-based system model and simulation model (see figure 5).

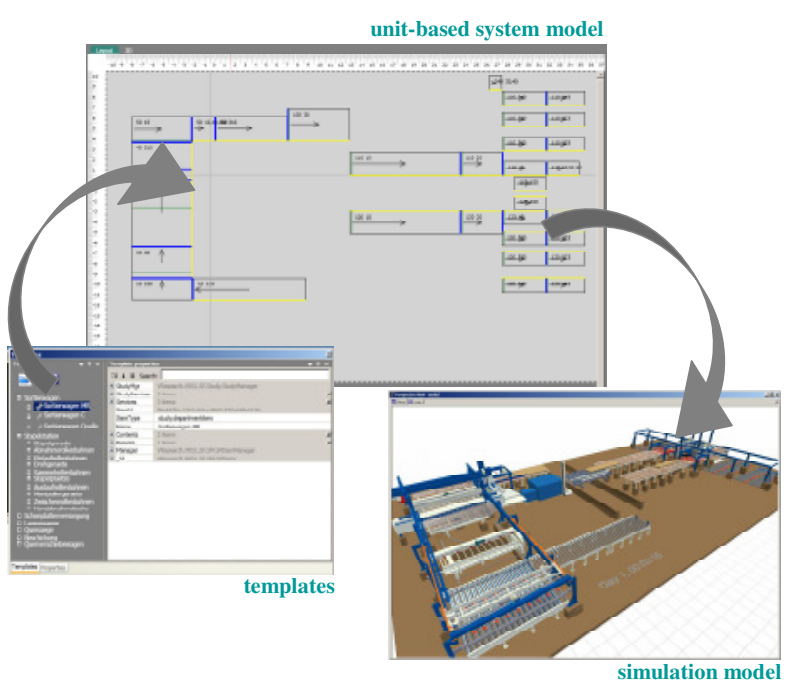

Figure 5 Simulation services

A plant unit template represents a self-contained plant unit, which consists of a set of devices. The collection of devices can be viewed with the template designer. A device is specified by attributes which describe processand mechanical information, velocities and metainformation for the instantiation of each class type used in the virtual field layer and the virtual control layer. They cannot be broken down further.

The graphical editor uses the previously described plant unit templates and allows the user to instantiate the plant units using drag-and-drop and to couple the material flows using a snap function. Once the model is specified as a unit-based system model it needs to be transformed into a simulation model. Therefore, the unit-based system model is persisted as a well-formed XML 
document. The transformation process to build a simulation model is automated and consists of three parts: The instantiation of the virtual control layer depending on the defined unit-based system model description, the creation of all simulation objects and the assignment of values to all public properties.

\section{Discrete event task handling}

The object-oriented hierarchical simulation model of the plant is based on the functional decomposition approach. The simulation includes the modeled units of the real plant and each unit of a production set is uniquely identifiable and traced during its lifecycle. The model is created according to the modeling process described in the previous section.

After starting the emulation the event list will be served through plant units of the virtual control layer. When the process control layer sends orders to designated plant units of the virtual control layer, all activities are registered as tasks and are stored in the event list in the correct order. The instructions set consists of 22 commands of three types:

- basic commands,

- motion commands and

- item operations.

Basic commands are generally used by the model to handle items. The command Move hands over a panel or part of it from a source to a destination plant unit. Motion commands describe the kinematic behavior of a plant unit or device. The instruction Travel moves a plant unit like a sorting carriage to a specified destination. Item operations relate to a panel, respectively parts of a panel. The instruction Create generates a stack of panel on a source plant unit like an infeed roller track.

In modern software systems it cannot be assumed that the sender of a message and the receiver of that message are located in the same address space. They may be executed on different processors or on different threads of the same processor (Douglass 2004). In case of control systems, each layer is located in a different thread. The communication mechanism between these layers is guarded by the blocking rendezvous pattern and is used to synchronize a set of threads or permit data sharing among a set of threads (Douglass 2004). In blocking rendezvous the sender waits for the receiver to accept and process the message.

The implementation of a blocking rendezvous pattern for handling the event list makes it possible to have a simulation model in which the behavior is independent of simulation speed. If the virtual field layer confirms the execution of a task sequence, the confirmation call is blocked until the process control layer has sent new orders to the destination plant units of the virtual control layer and all activities are registered as tasks and saved in the event list. The elapsed time up to the confirmation call is skipped and will not be counted towards simulation time. This is because in real-time, the system which is controlled, has no time delay between order confirmation and receipt of orders. Consequently, the connection between the simulation model and the process control layer allows logging of system states and measurement of the performance of the emulated system's cycle time.

\section{Emulation results and analysis}

While running the simulation or emulation model, results are written to an Oracle $(\subset$ database which afterwards can be viewed and analyzed by the user through the application platform. On the virtual field layer of the simulation system, two kinds of information are logged:

- The information whether a device is busy: The status of a device is busy if it is moving in some direction (turning, opening, closing, etc.).

- The information whether a plant unit is occupied: The status of a plant unit is occupied if a flow object is located on it.

With these two kinds of recorded information several sorts of result analysis can be carried out. These results are shown in tables and charts. The user has the possibility to group the displayed information by devices, plant units or regions of the plant units. Additionally it is possible to compare several simulation or emulation runs. With these options the user is able to analyze the utilization of plant units and devices (see figure 6).

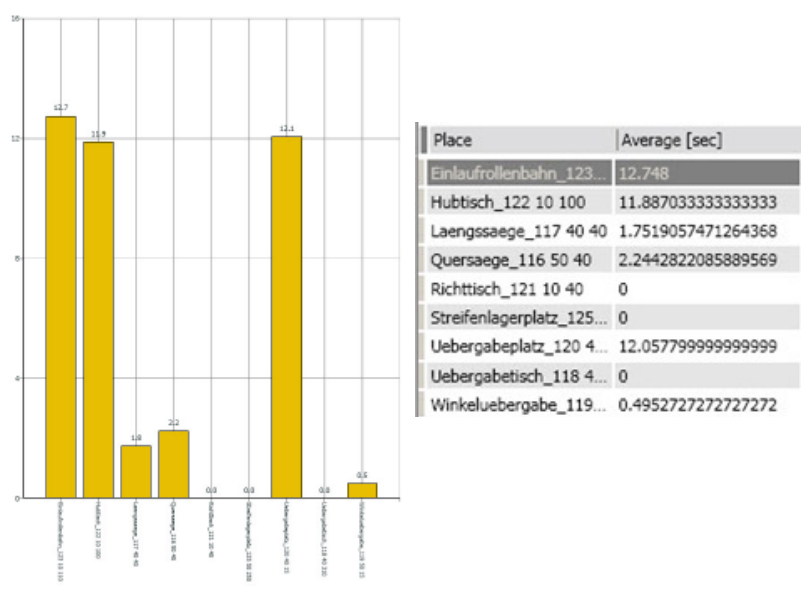

Figure 6 Analysis of utilization of plant units

Furthermore, throughput rates and cycle times of the cut-to-size plants can be viewed in detail (see figure 7). 


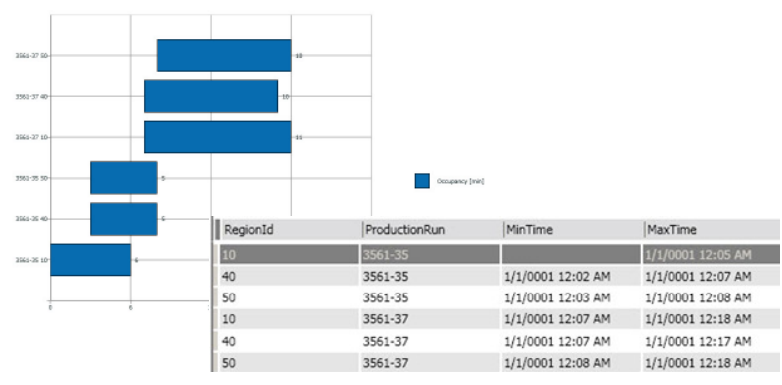

Figure 7 Analysis of cycle time of simulation runs

\section{RESULTS AND FINDINGS}

The implemented simulation and emulation tool provides the experts with relevant information, models and methods for the acquisition, the planning as well as the operation of cut-to-size plants. The tool facilitates the anticipation of the behavior of real plants on a virtual system and allows plant experts to model, simulate, emulate and animate sequences of cut-to-size plants without being experts in simulation or emulation. When building up an emulation model, plant units like sawing, sorting or stacking machines are put into a model and linked together according to the modeling process described in the previous chapters. By running the emulation job, data of the virtual machines are generated on the host system, processed in the emulation model and results are written to the database. Subsequently the analysis of cycle times, throughput and utilization of the plant units can be realized by the means of tables and charts.

The application of the decision support instrument brings various advantages in several phases of the selling and realization of cut-to-size plants (see figure 8).

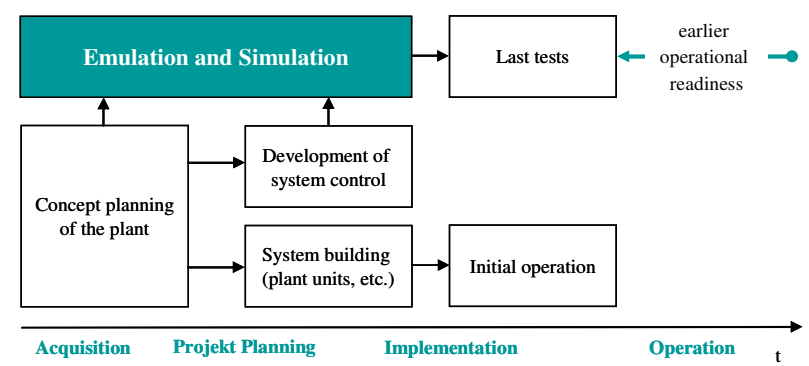

Figure 8 Plant engineering phases with emulation and animation

First of all, by using the decision support tool in the acquisition process vendors of cut-to-size plants can more easily demonstrate the plant concepts, which will help the customer to understand the facts. Especially the animation of material flow helps the user to get a clearer idea of the processes on cut-to-size plants. The possibility of calculating exact cycle times, throughput rates and utilizations of machines improves the accuracy of prediction of performance specifications. Therefore technical expertise, steadiness and reliability can be demonstrated through virtual plants. With this approach costs and time can be saved in the acquisition process. Apart from this, the tool supports the system experts in the plant planning process, in validation of control strategies and in the analysis of bottlenecks in the material flow. The comparison of several simulation scenarios is also possible and allows the system experts to constantly optimize the hardware and software. During implementation of the plant the tool can serve as training instrument to get familiar with the control system and plant processes. While operating the plant it is possible to perform impact analysis of modifications of the virtual as well as the real system. This allows an efficient analysis and resolution of errors without disrupting the normal course of business. With the realization of the described decision support tool a planning instrument could be developed which makes it possible to reduce acquisition and startup times for cutto-size plants and continuously test and optimize the processes.

Currently the operational use of the implemented simulation-based decision support instrument is being initiated in one company. The concerned specialist for cut-to-size plants was directly involved in the realization process of the simulation and emulation systems.

Additional activities in the further development and extension of the emulation tool are planned in course of this year. Currently the machine units can only be tested separately and not as a combined plant system. The idea is to create a complex system where a combination of reality and simulation (see figure 2) can be achieved. This would mean that real units of the plant could be tested within the whole virtual system. This approach will help the plant experts to be able to find failures on machines or in the material flow much earlier as without the decision support tool.

\section{REFERENCES}

Aleisa, E.E. and L. Lin. 2005. "For effectiveness facilities planning: Layout optimization then simulation, or vice versa?". In Proceedings of the 2005 Winter Simulation Conference.

Archibald, G.; N. Karabakal; and P. Karlsson. 1999. "Supply chain vs. supply chain: using simulation to compete beyond the four walls". In Proceedings of the 1999 Winter Simulation Conference, pp. 1207-1214.

Azadivar, F. and J. Wang. 2000. "Facility layout optimization using simulation and genetic algorithms". International Journal of Production Research, 38(17), 4369-4383.

Balzert H. 1999. "Lehrbuch der Objektmodellierung". Spektrum Akademischer Verlag, Heidelberg.

Banks, J.; S. Buckley; S. Jain; P. Lendermann; and M. Manivannan. 2002. "Panel session: opportunities for simulation in supply chain management". In Proceedings of the 2002 Winter Simulation Conference, 2, 1652-1658.

Banks, J.; J. S. Carson II; B. L. Nelson; D. M. Nicol. 2005. "Discrete-Event System Simulation". Fourth Edition, Pearson Prentice Hall. 
Chang, Y. and H. Makatsoris. 2001. "Supply chain modelling using simulation". International Journal of Simulation, 2 (1), 24-30.

Dobler, M.; M. Saler; and L. Maerz. 2008. "Distribution of Concurrent Simulation Runs on a Service-oriented Network Structure". Paper presented at the ASIM Conference 2008, Berlin, October 1 - 3.

Douglass B.P. 2004. "Real time UML: advances in the UML for real-time systems". Addison-Wesley.

Drake, G.R.; J.S. Smith; and B.A. Peters. 1995. "Simulation as a planning and scheduling tool for flexible manufacturing systems". In Proceedings of the 1995 Winter Simulation Conference, pp.805-812.

Guenthner, W. and M. ten Hompel. 2010. "Internet der Dinge in der Intralogistik“. Springer Verlag, Berlin.

Iannone, R.; S. Miranda; and S. Riemma. 2007. "Supply chain distributed simulation: An efficient architecture for multimodel synchronization". Simulation Modelling Practice and Theory, 15 (3), 221-236.

Irizarry, M.A.; J.R. Wilson; and J. Trevino. 2001. "A Flexible Simulation Tool for Manufacturing-cell Design". IIE Transactions 33(10), 827-836.

Kleijnen J. 2005. "Supply chain simulation tools and techniques: a survey". International Journal of Simulation \& Process Modelling, 1 (1/2), 82-89.

Maerz, L. and M. Saler. 2008: "An Analysis, Planning and Decision Tool for Warehouse Applications". Paper presented at the ASIM Conference 2008, Berlin, October 1 -3 .

Mc Gregor I. 2002. "The relationship between simulation and emulation". In the Proceedings of the 2002 Winter Simulation Conference, San Diego, California, USA:1683-1688.

Mertens P. 2001. Lexikon der Wirtschaftsinformatik, Springer Verlag, Berlin.

Morris, J.S. and R.J. Tersine. 1990. "A Simulation Analysis of Factors Influencing the Attractiveness of Group Technology Cellular Layouts". Management Science 36(12), 1567-1578.

Pekrul S. 2006. "Bauwirtschaft und Baubetrieb. Strategien und Maßnahmen zur Steigerung der Wettbewerbsfähigkeit deutscher Bauunternehmen“. Universitätsbibliothek, Berlin.

Min, H. and G. Zhou. 2002. "Supply chain modeling: past, present and future". Computers \& Industrial Engineering, 43 (1-2): 231-249.

Olhager, J. and F. Persson. 2006. "Simulating production and inventory control systems: a learning approach to operational excellence". Production Planning and Control, 17 (2), 113-127.

Phelps, R.; D. Parsons; and A. Siprelle. 2000. "The SDI Industry Product Suite: simulation from the production line to the supply chain". In the Proceedings of the 2000 Winter Simulation Conference, 208-214.

Phelps, R; D. Parsons; and A. Siprelle. 2001. "SDI Supply Chain Builder: simulation from atoms to the enterprise". In the Proceedings of the 2001 Winter Simulation Conference, 246-249.

Shah N. 2005. "Process industry supply chains: Advances and challenges". Computers and Chemical Engineering, 29 (6): $1225-1235$.

Soellner A. 2008. "Einfuehrung in das Internationale Management. Eine institutionenökonomische Perspektive“. Gabler GWV Fachverlage GmbH, Wiesbaden.
Siprelle, A.; D. Parsons; and R. Phelps. 1999. "SDI Industry Pro: simulation for enterprise-wide problem solving". In the Proceedings of the Winter Simulation Conference, 241-248.

Terzi, S. and S. Cavalieri. 2004. "Simulation in the supply chain context: a survey". Computers in Industry, 53 (1), 316.

VDI 3633. 2000. VDI-Richtlinien 3633, Simulation von Logistik-, Materialfluss- und Produktionssystemen, Grundlagen.

Zeigler, B. P. and H. S. Sarjoughian. 2003. "Introduction to DEVS Modeling and Simulation with JAVA: Developing Component-based Simulation Models". Arizona Center of Integrative Modeling and Simulation.

Zhou, M.C. and K. Venkatesh. 1999. "Modeling, simulation, and control of flexible manufacturing systems: a Petri net approach". World Scientific Publishing Company, Singapore.

Balci O. 1985. "Guidelines for Successful Simulation Studies". Technical report TR-85-2. Department of Computer Science, Virginia Tech, Blacksburg, Va. (Nov).

\section{AUTHOR BIOGRAPHIES}

V-Research is an Austrian competence center for industrial research and development. In the research field "Process Engineering for Construction, Manufacture and Logistics" the research center focuses on methods to support complex decision making in manufacturing and logistics processes.

Robert Schoech is project manager at V-Research. He develops systems focused on Simulation of industrial processes. His research interests also include track \& trace solutions based on GPS, GSM and RFID technology.

Susanne Schmid is research assistant and project manager at V-Research. Her main research areas include the conception, modeling and development of simulation models.

Christian Hillbrand is head of the research area „Technical Logistics” at V-Research and director of the research programme "ProDSS" (Integrated Decision Support Systems for Industrial Processes). He holds a $\mathrm{PhD}$-degree in business information systems from the University of Vienna. 